\title{
Estimation of Build-Up of Nuclide during Irradiation by Solving Ode through Adomian Decomposition Method (Adm).
}

\author{
Faweya, E.B ${ }^{1}$, Enoch, $\mathrm{O}^{2}$, Adesakin ,G.E ${ }^{3}$ and Faweya, $\mathrm{O}^{4}$ \\ 1,3 (Department of Physics, Faculty of Science/Ekiti State University, Nigeria) \\ 2,4 (Department of Mathematical Sciences, Faculty of Science/Ekiti State University, Nigeria)
}

\begin{abstract}
In this work, we considered first order differential equation (FODE) for nuclide build-up during irradiation of Gold ( $\mathrm{Au}$ ) with neutron. The approximate solutions to FODE are obtained using the Adomian Decomposition Method (ADM). Results have been found to be accurate and effective when compared with analytical solutions. Numerical computation was carried out using force. 3 software.
\end{abstract}

Keywords: Adomian, Build-up, Differential equation, Gold, Neutron.

\section{Introduction.}

Neutron activation analysis of elemental composition of various materials has been and continuous to be the subject of numerous researchers, since it is used to determine the concentration of trace and major elements of variety of materials. Examples of the types of materials that can be analyzed by NAA are cement, oil field, rocks, atmospheric aerosols, minerals, soils, archaeological artifacts, tree rings, dust in ice cores, hair, nails, refractory ores, skin, plant, animal matter and coal (Nargolwalla and Przybylowicz, 1973, Ellis, 1987, Ellis and Singer, 2008, Ioan, et al, 2008, Kogo, et al, 2009, waring, et al, 2011, Essiett, et al, 2011, Antoaneta, 2011, Nelson, 2012). The extensive use of NAA in environmental control and monitoring can be demonstrated by the large number of papers presented at the symposia organized by IAEA in these fields (Hassan, 2008) .These are due to the strengths of NAA which include ability to analyze large number of elements simultaneously, very low detections limits for many elements, small sample sizes (1-200 mg), no chemical preparation and non- destructive (Nelson, 2012). These works, though impressive and interesting have neglected the theoretical basis of this technique and stuck to practical cases. The decomposition methods has been shown to solve effectively, easily and accurately a large class of linear and non-linear, ordinary, partial (Evans and Raslan, 2004), non-stochastic (Such as FODE which is the basic NAA equation and frontier problem in Physics) or stochastic differential equations with approximate solutions that converge rapidly to accurate solutions (Adomian, G, 1994, Wazwaz, A.M, 2002, Abdusalam, H.A and Alabdulatif, M, 2006). These equations are found in wide fields of science, Engineering and Medicine. The main goal of the present research is to estimate nuclide build-up by solving the existing FODE (Utah, 2012) involve in this technique using Adomian Decomposition Method (ADM).

\subsection{Governing Equations}

In NAA, samples are activated by neutrons and these results in a nuclear reaction between neutrons and target nuclei (Molnar, 2012). During irradiation the naturally occurring stable isotopes of most elements that constitute the materials are transformed into radioactive isotopes by neutron capture. Then the activated nucleus decays according to a characteristic half-life; some nuclides emit particles only, but most nuclides emit gammaquanta too with specific energies (Molnar, 2012).

The equation governing these nuclear interactions is given by:

$N_{1}+n \rightarrow N_{2}$

1

where $N_{1}$ is the irradiated nuclide, $n$ is the neutron and $N_{2}$ is the number of nuclei present at any time $t$. The activity for a particular radionuclide at any time $t$ during irradiation is given by:

$A=N \sigma \varphi\left\lfloor 1-e^{-\lambda t_{i r r}}\right\rfloor$

equation 2 is the basic NAA equation (Nelson, 2012).

After a delay of time $t_{d}$, equation 2 becomes

$A=N \sigma \varphi\left[1-e^{-\lambda t_{i r r}}\right] e^{-\lambda t_{d}}$

And for counting time of $t_{c}$

$A=N \sigma \varphi\left[1-e^{-\lambda t_{i r r}}\right] e^{-\lambda t_{d}}\left[1-e^{-\lambda t_{c}}\right]$

Where $A\left(\mathrm{ds}^{-1}\right)$ is the activity, $N$ is the number of atoms of the target isotope, $\lambda\left(\mathrm{s}^{-1}\right)$ is the disintegration constant, $\varphi\left(\mathrm{ncm}^{-2} \mathrm{~s}^{-1}\right)$ is the neutron flux, $\sigma\left(10^{-24} \mathrm{~cm}^{2}\right)$ is the activation cross-section and $t_{i r}$ is the irradiation time.

Adapting the example of Utah, 2012 and for material balance for the nuclear reaction that took place when ${ }_{79}^{197} \mathrm{Au}$ is irradiated with neutron, the first order differential equation (FODE) obtained is as follows: 
$\frac{d N_{2}}{d t}=\varphi N_{1} \sigma_{a 1}-\left(\lambda_{2}+\varphi \sigma_{a 2}\right) N_{2}$

where $\sigma_{a 1}$ and $\sigma_{a 2}$ are cross-sections. Equation 5 was solved by Utah using the simplifying assumptions as follows:

(1) Effective removal cross-section-sum of the probability of the removal for the activated species by capture + capture

$\lambda_{2 T}=\lambda_{2}+\varphi \sigma_{a 2}$

6

(2) Unless the new species has a very large absorption cross-section and the fluxes are on the order of a power rector.

$\lambda_{2} \approx \lambda_{2 T}$

Rearrange equation 5 based on the aforementioned assumptions

$\frac{d N_{2}}{d t}+N_{2} \lambda_{2 T}=\varphi N_{1} \sigma_{a 1}$

Equation eight can be re-written as $\frac{d y}{d x}+a(x) y=h(x)$.

The solution to equation 8 by Utah using integrating factor $P=e^{\int a(x) d x}$ and applying boundary conditions $N_{2}=0$ at $t=0$ is given as

$N_{2}=\frac{\varphi N_{1} \sigma_{a 1}\left(1-e^{-\lambda} T^{t}\right)}{\lambda_{2 T}}$

Equation 9 represents the number of nuclei $\left(N_{2}\right)$ present at any time $t$ according to Utah (Utah, 2012).

\subsection{Simplifications Of Utah's Equations}

The first order differential equations that models equation 1 when Utah assumptions were used and neglected is as follows:

$\frac{d N_{2}}{d t}=\gamma-\beta N_{2} ; \quad N_{2}(0)=0 \quad$ (assumption neglected)

$\frac{d N_{2}}{d t}=\gamma-\lambda_{2 T} N_{2} ; N_{2}(0)=0 \quad$ (assumption used)

Equations 10 and 11 can indeed be solved by using integrating factor

$P=e^{\int p(x) d x}=e^{\int \beta d(t)}=e^{\beta t}$

$\left(\lambda_{2 T}+\varphi \sigma_{a 2}\right) t=e^{\lambda_{2 T} t+\varphi \sigma_{a 2} t}$

$e^{\lambda_{2 T}+\varphi \sigma_{a 2}} \frac{d N_{2}}{d t}+\beta e^{\beta t} N_{2}=\gamma e^{\beta t}$

$\frac{d}{d t}\left(e^{\beta t} N_{2}\right)=\gamma e^{\beta t}$

$\int \frac{d}{d t}\left(e^{\beta t} N_{2}\right) d t=\int \gamma e^{\beta t} d t$

$e^{\beta t} N_{2}=\frac{\gamma}{\beta} e^{\beta t}+C$

$N_{2}=\frac{\gamma}{\beta}+\frac{C}{e^{\beta t}}=\frac{\gamma}{\beta}+C e^{-\beta t}$

$N_{2}=\frac{\varphi N_{1} \sigma_{a 1}}{\lambda_{2 T}+\varphi \sigma_{a 2}}+C e^{\left(\lambda_{2 T}+\varphi \sigma_{a 2}\right) t}$

Imposing boundary conditions $N_{2}=0$ at $t=0$

$C=-\frac{\varphi N_{1} \sigma_{a 1}}{\lambda_{2 T}+\varphi \sigma_{a 2}}$

Then it follows that

$N_{2}=\frac{\varphi N_{1} \sigma_{a 1}}{\lambda_{2 T}+\varphi \sigma_{a 2}}\left(1-e^{-\left(\lambda_{2 T}+\varphi \sigma_{a 2}\right) t}\right)$

Equation 11 is equivalent of equation 7 by Utah.

\section{Solution Using Adomian Decomposition Method}

$\mathrm{ADM}$ was used here to solve differential equations 10 and 11 with initial and boundary conditions $N_{2}=0$ at $t=0$

$\frac{d N_{2}}{d t}=\gamma-\beta N_{2} ; N_{2}(0)=0$

Let $N_{2}=y$

We presented equations 10 and 11 in operator form

$\frac{d y}{d t}=\gamma-\beta y ; \quad y_{(0)}=0$

Let $L=\frac{d}{d t}$, then $L^{-1}$ will be a one fold integral, then

$L y=\gamma-\beta y$
where $L$ is the linear operator and applying the inverse operator on 14 , we have

$$
\sum_{n=0}^{\infty} y_{n}(x)=L^{-1}(\gamma-\beta y)+y_{0}
$$




$$
\begin{gathered}
\sum_{n=0}^{\infty} y_{n}(x)=\int_{0}^{t} \gamma d t-\int_{0}^{t} \beta y_{n} d t+y_{0} \\
\sum_{n=0}^{\infty} y_{n}(x)=y_{0}+\left(\gamma t-\beta \int_{0}^{t} y d t\right)
\end{gathered}
$$

Based on the Decomposition method, the solution to $y_{n} x$ be as follows for five (5) steps.

$$
\begin{aligned}
& y_{0}(x)=y_{0} \\
& y_{n+1}(x)=\left(\gamma t-\beta \int_{0}^{t} y_{n} d t\right) ; \quad n \geq 0 \\
& y_{1}(x)=\left(\gamma t-\beta \int_{0}^{t} y_{o} d t\right)=\gamma t-\beta \int_{0}^{t}(0) d t=\gamma t \\
& y_{2}(x)=\left(\gamma t-\beta \int_{0}^{t} y_{1} d t\right)=\gamma t-\frac{\beta \gamma t^{2}}{2} \\
& y_{3}(x)=\left(\gamma t-\beta \int_{0}^{t} y_{2} d t\right)=\gamma t-\beta \int_{0}^{t}\left(\gamma t-\frac{\beta \gamma t^{2}}{2}\right) d t=\gamma t-\beta\left(\frac{\gamma t^{2}}{2}-\frac{\beta \gamma t^{3}}{6}\right)=\gamma t-\frac{\beta \gamma t^{2}}{2}+\frac{\beta^{2} \gamma t^{3}}{6} \\
& y_{4}(x)=\int_{0}^{t}\left(\gamma-\beta y_{3}\right) d t=\gamma t-\beta \int_{0}^{t} y_{3} d t=\gamma t-\beta \int_{0}^{t}\left(\gamma t-\frac{\beta \gamma t^{2}}{2}+\frac{\beta^{2} \gamma t^{3}}{6}\right) d t=\gamma t-\frac{\beta \gamma t^{2}}{2}+\frac{\beta^{2} \gamma t^{3}}{6}-\frac{\beta^{3} \gamma t^{4}}{24} \\
& y_{5}(x)=\int_{0}^{t}\left(\gamma-\beta y_{4}\right) d t=\gamma t-\beta \int_{0}^{t}\left(\gamma t-\frac{\beta \gamma t^{2}}{2}+\frac{\beta^{2} \gamma t^{3}}{6}+\frac{\beta^{3} \gamma t^{4}}{24}\right) d t=\gamma t-\frac{\beta \gamma t^{2}}{2}+\frac{\beta^{2} \gamma t^{3}}{6}-\frac{\beta^{3} \gamma t^{4}}{24}+\frac{\beta^{4} \gamma t^{5}}{120} \\
& y_{n}(x)=\gamma t-\frac{\beta \gamma t^{2}}{2 !}+\frac{\beta^{2} \gamma t^{3}}{3 !}-\frac{\beta^{3} \gamma t^{4}}{4 !}+\cdots \ldots \ldots+\frac{\beta^{n-1} \gamma t^{n}}{n !} \\
& \sum_{n=0}^{\infty} y_{n}(x)=y_{0}(x)+y_{1}(x)+y_{2}(x)+y_{3}(x)+y_{4}(x)+y_{5}(x)+\cdots \cdot y_{n}(x) \\
& =0+\gamma t+\gamma t-\frac{\beta \gamma t^{2}}{2 !}+\gamma t-\frac{\beta \gamma t^{2}}{2 !}+\frac{\beta^{2} \gamma t^{3}}{3 !}+\gamma t-\frac{\beta \gamma t^{2}}{2 !}+\frac{\beta^{2} \gamma t^{3}}{3 !}-\frac{\beta^{3} \gamma t^{4}}{4 !}+\gamma t-\frac{\beta \gamma t^{2}}{2 !}+\frac{\beta^{2} \gamma t^{3}}{3 !}-\frac{\beta^{3} \gamma t^{4}}{4 !}+\frac{\beta^{4} \gamma t^{5}}{5 !}+ \\
& =5 \gamma t-\frac{4 \beta \gamma t^{2}}{2 !}+\frac{3 \beta^{2} \gamma t^{3}}{3 !}-\frac{2 \beta^{3} \gamma t^{4}}{4 !} \\
& \begin{aligned}
\sum_{n=0}^{k} y_{n}(x)=(k- & n)(n-1)^{n}\left[\frac{\beta^{n} \gamma t^{n-1}}{n !}\right] \\
& =(k-n)(-1)^{n}\left[\frac{\beta^{n} \gamma t^{n-1}}{n !}\right] \operatorname{or}(k+1-n)(-1)^{n}\left[\frac{\beta^{n-1} \gamma t^{n}}{n !}\right]
\end{aligned}
\end{aligned}
$$

We now used Force. 3 to compute for both Adomian and Utah's equation using 1 day at a time and 2.7 days (half-life) in the Utah's data as shown in Table 1.

Table 1: Utah's data for ${ }_{79}^{197} \mathrm{Au}$ during irradiation by neutron (Utah, 2012)

\begin{tabular}{|c|c|c|c|c|}
\hline Diameter (cm) & Thickness $(\mathbf{m m})$ & Flux $\left.\mathbf{( n / \mathbf { c m } ^ { 2 }} \mathbf{s}\right)$ & $\square_{\mathbf{a 1}}(\mathbf{b a r n s})$ & $\square_{\mathbf{a 2}}$ (barns) \\
2.50 & 1.00 & $10^{8}$ & 98.8 & 26000 \\
\hline
\end{tabular}

We took hundreds and thirty terms of the series for fractionated days and one day at a time to show numerically whether the ADM leads to higher accuracy. The results obtained using force. 3 for Adomian and Utah (Actual) is presented in Tables 2 to 5. We then compared between the present scheme and the actual. This shows that the errors are infinitesimal. The solutions of $N_{2}$ from ADM and actual are plotted in figures 1to 4. These figures superimposed, this confirms the accuracy and reliability of ADM.

$\mathrm{BET}=0.2567$

Table 2: Result for a day at a time we used Utah's assumptions.

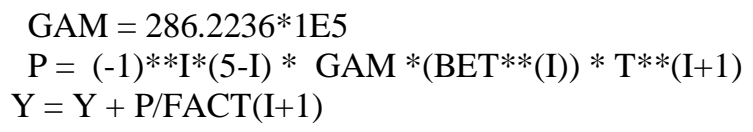

$\mathrm{H}=1.0$. That is a day at a time.

\begin{tabular}{|l|l|l|l|}
\hline $\mathbf{X}$ & ADOMIAN & ACTUAL & ERROR \\
\hline 0.0 & $0.00000000 \mathrm{E}+00$ & $0.00000000 \mathrm{E}+00$ & $0.00000000 \mathrm{E}+00$ \\
\hline 1.0 & $0.25243843 \mathrm{E}+09$ & $0.25243843 \mathrm{E}+09$ & $0.00000000 \mathrm{E}+00$ \\
\hline 2.0 & $0.44772493 \mathrm{E}+09$ & $0.44772493 \mathrm{E}+09$ & $0.00000000 \mathrm{E}+00$ \\
\hline
\end{tabular}


Estimation Of Build-Up Of Nuclide During Irradiation By Solving Ode Through Adomian

\begin{tabular}{|l|l|l|l|}
\hline 3.0 & $0.59879853 \mathrm{E}+09$ & $0.59879853 \mathrm{E}+09$ & $0.00000000 \mathrm{E}+00$ \\
\hline 4.0 & $0.71566918 \mathrm{E}+09$ & $0.71566918 \mathrm{E}+09$ & $0.00000000 \mathrm{E}+00$ \\
\hline 5.0 & $0.80608032 \mathrm{E}+09$ & $0.80608032 \mathrm{E}+09$ & $0.00000000 \mathrm{E}+00$ \\
\hline 6.0 & $0.87602240 \mathrm{E}+09$ & $0.87602240 \mathrm{E}+09$ & $0.00000000 \mathrm{E}+00$ \\
\hline 7.0 & $0.93012954 \mathrm{E}+09$ & $0.93012954 \mathrm{E}+09$ & $0.00000000 \mathrm{E}+00$ \\
\hline 8.0 & $0.97198694 \mathrm{E}+09$ & $0.97198694 \mathrm{E}+09$ & $0.00000000 \mathrm{E}+00$ \\
\hline 9.0 & $0.10043678 \mathrm{E}+10$ & $0.10043678 \mathrm{E}+10$ & $0.00000000 \mathrm{E}+00$ \\
\hline 10.0 & $0.10294177 \mathrm{E}+10$ & $0.10294177 \mathrm{E}+10$ & $0.00000000 \mathrm{E}+00$ \\
\hline 11.0 & $0.10487963 \mathrm{E}+10$ & $0.10487962 \mathrm{E}+10$ & $0.64000000 \mathrm{E}+02$ \\
\hline 12.0 & $0.10637874 \mathrm{E}+10$ & $0.10637875 \mathrm{E}+10$ & $0.64000000 \mathrm{E}+02$ \\
\hline 13.0 & $0.10753847 \mathrm{E}+10$ & $0.10753847 \mathrm{E}+10$ & $0.00000000 \mathrm{E}+00$ \\
\hline 14.0 & $0.10843564 \mathrm{E}+10$ & $0.10843564 \mathrm{E}+10$ & $0.00000000 \mathrm{E}+00$ \\
\hline 15.0 & $0.10912968 \mathrm{E}+10$ & $0.10912968 \mathrm{E}+10$ & $0.00000000 \mathrm{E}+00$ \\
\hline 16.0 & $0.10966659 \mathrm{E}+10$ & $0.10966659 \mathrm{E}+10$ & $0.00000000 \mathrm{E}+00$ \\
\hline 17.0 & $0.11008195 \mathrm{E}+10$ & $0.11008195 \mathrm{E}+10$ & $0.00000000 \mathrm{E}+00$ \\
\hline 18.0 & $0.11040328 \mathrm{E}+10$ & $0.11040328 \mathrm{E}+10$ & $0.00000000 \mathrm{E}+00$ \\
\hline 19.0 & $0.11065184 \mathrm{E}+10$ & $0.11065184 \mathrm{E}+10$ & $0.00000000 \mathrm{E}+00$ \\
\hline 20.0 & $0.11084415 \mathrm{E}+10$ & $0.11084413 \mathrm{E}+10$ & $0.12800000 \mathrm{E}+03$ \\
\hline 21.0 & $0.11099290 \mathrm{E}+10$ & $0.11099290 \mathrm{E}+10$ & $0.00000000 \mathrm{E}+00$ \\
\hline 22.0 & $0.11110797 \mathrm{E}+10$ & $0.11110798 \mathrm{E}+10$ & $0.12800000 \mathrm{E}+03$ \\
\hline 23.0 & $0.11119698 \mathrm{E}+10$ & $0.11119700 \mathrm{E}+10$ & $0.25600000 \mathrm{E}+03$ \\
\hline 24.0 & $0.11126589 \mathrm{E}+10$ & $0.11126588 \mathrm{E}+10$ & $0.12800000 \mathrm{E}+03$ \\
\hline 25.0 & $0.11131914 \mathrm{E}+10$ & $0.11131916 \mathrm{E}+10$ & $0.12800000 \mathrm{E}+03$ \\
\hline 26.0 & $0.11136036 \mathrm{E}+10$ & $0.11136037 \mathrm{E}+10$ & $0.12800000 \mathrm{E}+03$ \\
\hline 27.0 & $0.11139226 \mathrm{E}+10$ & $0.11139226 \mathrm{E}+10$ & $0.00000000 \mathrm{E}+00$ \\
\hline 28.0 & $0.11141693 \mathrm{E}+10$ & $0.11141692 \mathrm{E}+10$ & $0.12800000 \mathrm{E}+03$ \\
\hline 29.0 & $0.11143598 \mathrm{E}+10$ & $0.11143601 \mathrm{E}+10$ & $0.25600000 \mathrm{E}+03$ \\
\hline 30.0 & $0.11145073 \mathrm{E}+10$ & $0.11145076 \mathrm{E}+10$ & $0.38400000 \mathrm{E}+03$ \\
\hline & & & \\
\hline
\end{tabular}

Table 3: Result when we used Utah's assumptions for fractionated days (2.7 days).

\begin{tabular}{|c|c|c|c|}
\hline $\mathbf{X}$ & ADOMIAN & ACTUAL & ERROR \\
\hline 0.0 & $0.00000000 \mathrm{E}+00$ & $0.00000000 \mathrm{E}+00$ & $0.00000000 \mathrm{E}+00$ \\
\hline 0.3 & $0.74663112 \mathrm{E}+08$ & $0.74663112 \mathrm{E}+08$ & $0.00000000 \mathrm{E}+00$ \\
\hline 0.5 & $0.14432658 \mathrm{E}+09$ & $0.14432658 \mathrm{E}+09$ & $0.00000000 \mathrm{E}+00$ \\
\hline 0.8 & $0.20932536 \mathrm{E}+09$ & $0.20932536 \mathrm{E}+09$ & $0.00000000 \mathrm{E}+00$ \\
\hline 1.1 & $0.26997162 \mathrm{E}+09$ & $0.26997162 \mathrm{E}+09$ & $0.00000000 \mathrm{E}+00$ \\
\hline 1.4 & $0.32655696 \mathrm{E}+09$ & $0.32655696 \mathrm{E}+09$ & $0.00000000 \mathrm{E}+00$ \\
\hline 1.6 & $0.37935325 \mathrm{E}+09$ & $0.37935325 \mathrm{E}+09$ & $0.00000000 \mathrm{E}+00$ \\
\hline 1.9 & $0.42861418 \mathrm{E}+09$ & $0.42861418 \mathrm{E}+09$ & $0.00000000 \mathrm{E}+00$ \\
\hline 2.2 & $0.47457651 \mathrm{E}+09$ & $0.47457651 \mathrm{E}+09$ & $0.00000000 \mathrm{E}+00$ \\
\hline 2.4 & $0.51746115 \mathrm{E}+09$ & $0.51746115 \mathrm{E}+09$ & $0.00000000 \mathrm{E}+00$ \\
\hline 2.7 & $0.55747424 \mathrm{E}+09$ & $0.55747424 \mathrm{E}+09$ & $0.00000000 \mathrm{E}+00$ \\
\hline 3.0 & $0.59480787 \mathrm{E}+09$ & $0.59480787 \mathrm{E}+09$ & $0.00000000 \mathrm{E}+00$ \\
\hline 3.2 & $0.62964160 \mathrm{E}+09$ & $0.62964160 \mathrm{E}+09$ & $0.00000000 \mathrm{E}+00$ \\
\hline 3.5 & $0.66214278 \mathrm{E}+09$ & $0.66214278 \mathrm{E}+09$ & $0.00000000 \mathrm{E}+00$ \\
\hline 3.8 & $0.69246771 \mathrm{E}+09$ & $0.69246771 \mathrm{E}+09$ & $0.00000000 \mathrm{E}+00$ \\
\hline 4.1 & $0.72076198 \mathrm{E}+09$ & $0.72076198 \mathrm{E}+09$ & $0.00000000 \mathrm{E}+00$ \\
\hline 4.3 & $0.74716166 \mathrm{E}+09$ & $0.74716166 \mathrm{E}+09$ & $0.00000000 \mathrm{E}+00$ \\
\hline 4.6 & $0.77179347 \mathrm{E}+09$ & $0.77179347 \mathrm{E}+09$ & $0.00000000 \mathrm{E}+00$ \\
\hline 4.9 & $0.79477600 \mathrm{E}+09$ & $0.79477600 \mathrm{E}+09$ & $0.00000000 \mathrm{E}+00$ \\
\hline 5.1 & $0.81621952 \mathrm{E}+09$ & $0.81621952 \mathrm{E}+09$ & $0.00000000 \mathrm{E}+00$ \\
\hline 5.4 & $0.83622720 \mathrm{E}+09$ & $0.83622720 \mathrm{E}+09$ & $0.00000000 \mathrm{E}+00$ \\
\hline 5.7 & $0.85489510 \mathrm{E}+09$ & $0.85489510 \mathrm{E}+09$ & $0.00000000 \mathrm{E}+00$ \\
\hline 5.9 & $0.87231296 \mathrm{E}+09$ & $0.87231296 \mathrm{E}+09$ & $0.00000000 \mathrm{E}+00$ \\
\hline 6.2 & $0.88856454 \mathrm{E}+09$ & $0.88856454 \mathrm{E}+09$ & $0.00000000 \mathrm{E}+00$ \\
\hline 6.5 & $0.90372781 \mathrm{E}+09$ & $0.90372781 \mathrm{E}+09$ & $0.00000000 \mathrm{E}+00$ \\
\hline 6.8 & $0.91787584 \mathrm{E}+09$ & $0.91787584 \mathrm{E}+09$ & $0.00000000 \mathrm{E}+00$ \\
\hline 7.0 & $0.93107635 \mathrm{E}+09$ & $0.93107635 \mathrm{E}+09$ & $0.00000000 \mathrm{E}+00$ \\
\hline 7.3 & $0.94339296 \mathrm{E}+09$ & $0.94339296 \mathrm{E}+09$ & $0.00000000 \mathrm{E}+00$ \\
\hline 7.6 & $0.95488486 \mathrm{E}+09$ & $0.95488486 \mathrm{E}+09$ & $0.00000000 \mathrm{E}+00$ \\
\hline 7.8 & $0.96560730 \mathrm{E}+09$ & $0.96560730 \mathrm{E}+09$ & $0.00000000 \mathrm{E}+00$ \\
\hline 8.1 & $0.97561165 \mathrm{E}+09$ & $0.97561165 \mathrm{E}+09$ & $0.00000000 \mathrm{E}+00$ \\
\hline 8.4 & $0.98494624 \mathrm{E}+09$ & $0.98494624 \mathrm{E}+09$ & $0.00000000 \mathrm{E}+00$ \\
\hline
\end{tabular}


Estimation Of Build-Up Of Nuclide During Irradiation By Solving Ode Through Adomian

\begin{tabular}{|c|c|c|c|}
\hline 8.6 & $0.99365568 \mathrm{E}+09$ & $0.99365568 \mathrm{E}+09$ & $0.00000000 \mathrm{E}+00$ \\
\hline 8.9 & $0.10017819 \mathrm{E}+10$ & $0.10017819 \mathrm{E}+10$ & $0.00000000 \mathrm{E}+00$ \\
\hline 9.2 & $0.10093640 \mathrm{E}+10$ & $0.10093640 \mathrm{E}+10$ & $0.00000000 \mathrm{E}+00$ \\
\hline 9.5 & $0.10164384 \mathrm{E}+10$ & $0.10164384 \mathrm{E}+10$ & $0.00000000 \mathrm{E}+00$ \\
\hline 9.7 & $0.10230390 \mathrm{E}+10$ & $0.10230390 \mathrm{E}+10$ & $0.00000000 \mathrm{E}+00$ \\
\hline 10.0 & $0.10291978 \mathrm{E}+10$ & $0.10291978 \mathrm{E}+10$ & $0.00000000 \mathrm{E}+00$ \\
\hline 10.3 & $0.10349440 \mathrm{E}+10$ & $0.10349440 \mathrm{E}+10$ & $0.00000000 \mathrm{E}+00$ \\
\hline 10.5 & $0.10403055 \mathrm{E}+10$ & $0.10403055 \mathrm{E}+10$ & $0.00000000 \mathrm{E}+00$ \\
\hline 10.8 & $0.10453080 \mathrm{E}+10$ & $0.10453080 \mathrm{E}+10$ & $0.00000000 \mathrm{E}+00$ \\
\hline 11.1 & $0.10499754 \mathrm{E}+10$ & $0.10499755 \mathrm{E}+10$ & $0.64000000 \mathrm{E}+02$ \\
\hline 11.3 & $0.10543305 \mathrm{E}+10$ & $0.10543305 \mathrm{E}+10$ & $0.00000000 \mathrm{E}+00$ \\
\hline 11.6 & $0.10583939 \mathrm{E}+10$ & $0.10583939 \mathrm{E}+10$ & $0.00000000 \mathrm{E}+00$ \\
\hline 11.9 & $0.10621851 \mathrm{E}+10$ & $0.10621851 \mathrm{E}+10$ & $0.00000000 \mathrm{E}+00$ \\
\hline 12.2 & $0.10657224 \mathrm{E}+10$ & $0.10657224 \mathrm{E}+10$ & $0.00000000 \mathrm{E}+00$ \\
\hline 12.4 & $0.10690230 \mathrm{E}+10$ & $0.10690230 \mathrm{E}+10$ & $0.00000000 \mathrm{E}+00$ \\
\hline 12.7 & $0.10721025 \mathrm{E}+10$ & $0.10721025 \mathrm{E}+10$ & $0.00000000 \mathrm{E}+00$ \\
\hline 13.0 & $0.10749757 \mathrm{E}+10$ & $0.10749757 \mathrm{E}+10$ & $0.00000000 \mathrm{E}+00$ \\
\hline 13.2 & $0.10776567 \mathrm{E}+10$ & $0.10776567 \mathrm{E}+10$ & $0.00000000 \mathrm{E}+00$ \\
\hline 13.5 & $0.10801581 \mathrm{E}+10$ & $0.10801581 \mathrm{E}+10$ & $0.00000000 \mathrm{E}+00$ \\
\hline 13.8 & $0.10824920 \mathrm{E}+10$ & $0.10824920 \mathrm{E}+10$ & $0.00000000 \mathrm{E}+00$ \\
\hline 14.0 & $0.10846696 \mathrm{E}+10$ & $0.10846696 \mathrm{E}+10$ & $0.00000000 \mathrm{E}+00$ \\
\hline 14.3 & $0.10867013 \mathrm{E}+10$ & $0.10867013 \mathrm{E}+10$ & $0.00000000 \mathrm{E}+00$ \\
\hline 14.6 & $0.10885971 \mathrm{E}+10$ & $0.10885971 \mathrm{E}+10$ & $0.00000000 \mathrm{E}+00$ \\
\hline 14.9 & $0.10903660 \mathrm{E}+10$ & $0.10903660 \mathrm{E}+10$ & $0.00000000 \mathrm{E}+00$ \\
\hline 15.1 & $0.10920163 \mathrm{E}+10$ & $0.10920163 \mathrm{E}+10$ & $0.00000000 \mathrm{E}+00$ \\
\hline 15.4 & $0.10935561 \mathrm{E}+10$ & $0.10935561 \mathrm{E}+10$ & $0.00000000 \mathrm{E}+00$ \\
\hline 15.7 & $0.10949929 \mathrm{E}+10$ & $0.10949929 \mathrm{E}+10$ & $0.00000000 \mathrm{E}+00$ \\
\hline 15.9 & $0.10963333 \mathrm{E}+10$ & $0.10963333 \mathrm{E}+10$ & $0.00000000 \mathrm{E}+00$ \\
\hline 16.2 & $0.10975841 \mathrm{E}+10$ & $0.10975841 \mathrm{E}+10$ & $0.00000000 \mathrm{E}+00$ \\
\hline 16.5 & $0.10987511 \mathrm{E}+10$ & $0.10987511 \mathrm{E}+10$ & $0.00000000 \mathrm{E}+00$ \\
\hline 16.7 & $0.10998400 \mathrm{E}+10$ & $0.10998400 \mathrm{E}+10$ & $0.00000000 \mathrm{E}+00$ \\
\hline 17.0 & $0.11008558 \mathrm{E}+10$ & $0.11008559 \mathrm{E}+10$ & $0.12800000 \mathrm{E}+03$ \\
\hline 17.3 & $0.11018040 \mathrm{E}+10$ & $0.11018039 \mathrm{E}+10$ & $0.12800000 \mathrm{E}+03$ \\
\hline 17.6 & $0.11026883 \mathrm{E}+10$ & $0.11026883 \mathrm{E}+10$ & $0.00000000 \mathrm{E}+00$ \\
\hline 17.8 & $0.11035136 \mathrm{E}+10$ & $0.11035136 \mathrm{E}+10$ & $0.00000000 \mathrm{E}+00$ \\
\hline 18.1 & $0.11042836 \mathrm{E}+10$ & $0.11042835 \mathrm{E}+10$ & $0.12800000 \mathrm{E}+03$ \\
\hline 18.4 & $0.11050017 \mathrm{E}+10$ & $0.11050019 \mathrm{E}+10$ & $0.12800000 \mathrm{E}+03$ \\
\hline 18.6 & $0.11056721 \mathrm{E}+10$ & $0.11056722 \mathrm{E}+10$ & $0.12800000 \mathrm{E}+03$ \\
\hline 18.9 & $0.11062977 \mathrm{E}+10$ & $0.11062976 \mathrm{E}+10$ & $0.12800000 \mathrm{E}+03$ \\
\hline 19.2 & $0.11068812 \mathrm{E}+10$ & $0.11068812 \mathrm{E}+10$ & $0.00000000 \mathrm{E}+00$ \\
\hline 19.4 & $0.11074257 \mathrm{E}+10$ & $0.11074257 \mathrm{E}+10$ & $0.00000000 \mathrm{E}+00$ \\
\hline 19.7 & $0.11079337 \mathrm{E}+10$ & $0.11079337 \mathrm{E}+10$ & $0.00000000 \mathrm{E}+00$ \\
\hline 20.0 & $0.11084076 \mathrm{E}+10$ & $0.11084076 \mathrm{E}+10$ & $0.00000000 \mathrm{E}+00$ \\
\hline 20.3 & $0.11088499 \mathrm{E}+10$ & $0.11088499 \mathrm{E}+10$ & $0.00000000 \mathrm{E}+00$ \\
\hline 20.5 & $0.11092623 \mathrm{E}+10$ & $0.11092625 \mathrm{E}+10$ & $0.12800000 \mathrm{E}+03$ \\
\hline 20.8 & $0.11096476 \mathrm{E}+10$ & $0.11096475 \mathrm{E}+10$ & $0.12800000 \mathrm{E}+03$ \\
\hline 21.1 & $0.11100068 \mathrm{E}+10$ & $0.11100068 \mathrm{E}+10$ & $0.00000000 \mathrm{E}+00$ \\
\hline 21.3 & $0.11103419 \mathrm{E}+10$ & $0.11103419 \mathrm{E}+10$ & $0.00000000 \mathrm{E}+00$ \\
\hline 21.6 & $0.11106547 \mathrm{E}+10$ & $0.11106546 \mathrm{E}+10$ & $0.12800000 \mathrm{E}+03$ \\
\hline 21.9 & $0.11109466 \mathrm{E}+10$ & $0.11109464 \mathrm{E}+10$ & $0.12800000 \mathrm{E}+03$ \\
\hline 22.1 & $0.11112184 \mathrm{E}+10$ & $0.11112187 \mathrm{E}+10$ & $0.25600000 \mathrm{E}+03$ \\
\hline 22.4 & $0.11114729 \mathrm{E}+10$ & $0.11114726 \mathrm{E}+10$ & $0.25600000 \mathrm{E}+03$ \\
\hline 22.7 & $0.11117097 \mathrm{E}+10$ & $0.11117097 \mathrm{E}+10$ & $0.00000000 \mathrm{E}+00$ \\
\hline 23.0 & $0.11119306 \mathrm{E}+10$ & $0.11119308 \mathrm{E}+10$ & $0.12800000 \mathrm{E}+03$ \\
\hline 23.2 & $0.11121372 \mathrm{E}+10$ & $0.11121371 \mathrm{E}+10$ & $0.12800000 \mathrm{E}+03$ \\
\hline 23.5 & $0.11123297 \mathrm{E}+10$ & $0.11123296 \mathrm{E}+10$ & $0.12800000 \mathrm{E}+03$ \\
\hline 23.8 & $0.11125094 \mathrm{E}+10$ & $0.11125093 \mathrm{E}+10$ & $0.12800000 \mathrm{E}+03$ \\
\hline 24.0 & $0.11126769 \mathrm{E}+10$ & $0.11126769 \mathrm{E}+10$ & $0.00000000 \mathrm{E}+00$ \\
\hline 24.3 & $0.11128330 \mathrm{E}+10$ & $0.11128332 \mathrm{E}+10$ & $0.12800000 \mathrm{E}+03$ \\
\hline 24.6 & $0.11129789 \mathrm{E}+10$ & $0.11129792 \mathrm{E}+10$ & $0.25600000 \mathrm{E}+03$ \\
\hline 24.8 & $0.11131151 \mathrm{E}+10$ & $0.11131153 \mathrm{E}+10$ & $0.12800000 \mathrm{E}+03$ \\
\hline 25.1 & $0.11132421 \mathrm{E}+10$ & $0.11132422 \mathrm{E}+10$ & $0.12800000 \mathrm{E}+03$ \\
\hline 25.4 & $0.11133609 \mathrm{E}+10$ & $0.11133608 \mathrm{E}+10$ & $0.12800000 \mathrm{E}+03$ \\
\hline
\end{tabular}


Estimation Of Build-Up Of Nuclide During Irradiation By Solving Ode Through Adomian

\begin{tabular}{|l|l|l|l|}
\hline 25.7 & $0.11134710 \mathrm{E}+10$ & $0.11134714 \mathrm{E}+10$ & $0.38400000 \mathrm{E}+03$ \\
\hline 25.9 & $0.11135744 \mathrm{E}+10$ & $0.11135745 \mathrm{E}+10$ & $0.12800000 \mathrm{E}+03$ \\
\hline 26.2 & $0.11136712 \mathrm{E}+10$ & $0.11136708 \mathrm{E}+10$ & $0.38400000 \mathrm{E}+03$ \\
\hline 26.5 & $0.11137608 \mathrm{E}+10$ & $0.11137606 \mathrm{E}+10$ & $0.12800000 \mathrm{E}+03$ \\
\hline 26.7 & $0.11138447 \mathrm{E}+10$ & $0.11138444 \mathrm{E}+10$ & $0.38400000 \mathrm{E}+03$ \\
\hline 27.0 & $0.11139226 \mathrm{E}+10$ & $0.11139226 \mathrm{E}+10$ & $0.00000000 \mathrm{E}+00$ \\
\hline
\end{tabular}

Table 4: Result when Utah's assumptions are ignored for fractionated days (2.7 days)

\begin{tabular}{|c|c|c|c|}
\hline $\bar{X}$ & ADOMIAN & ACTUAL & ERROR \\
\hline 0.0 & $0.00000000 \mathrm{E}+00$ & $0.00000000 \mathrm{E}+00$ & $0.00000000 \mathrm{E}+00$ \\
\hline 0.3 & $0.74659400 \mathrm{E}+08$ & $0.74663112 \mathrm{E}+08$ & $0.37120000 \mathrm{E}+04$ \\
\hline 0.5 & $0.14431946 \mathrm{E}+09$ & $0.14432658 \mathrm{E}+09$ & $0.71200000 \mathrm{E}+04$ \\
\hline 0.8 & $0.20931510 \mathrm{E}+09$ & $0.20932536 \mathrm{E}+09$ & $0.10256000 \mathrm{E}+05$ \\
\hline 1.1 & $0.26995846 \mathrm{E}+09$ & $0.26997162 \mathrm{E}+09$ & $0.13152000 \mathrm{E}+05$ \\
\hline 1.4 & $0.32654115 \mathrm{E}+09$ & $0.32655696 \mathrm{E}+09$ & $0.15808000 \mathrm{E}+05$ \\
\hline 1.6 & $0.37933501 \mathrm{E}+09$ & $0.37935325 \mathrm{E}+09$ & $0.18240000 \mathrm{E}+05$ \\
\hline 1.9 & $0.42859370 \mathrm{E}+09$ & $0.42861418 \mathrm{E}+09$ & $0.20480000 \mathrm{E}+05$ \\
\hline 2.2 & $0.47455398 \mathrm{E}+09$ & $0.47457651 \mathrm{E}+09$ & $0.22528000 \mathrm{E}+05$ \\
\hline 2.4 & $0.51743670 \mathrm{E}+09$ & $0.51746115 \mathrm{E}+09$ & $0.24448000 \mathrm{E}+05$ \\
\hline 2.7 & $0.55744806 \mathrm{E}+09$ & $0.55747424 \mathrm{E}+09$ & $0.26176000 \mathrm{E}+05$ \\
\hline 3.0 & $0.59478010 \mathrm{E}+09$ & $0.59480787 \mathrm{E}+09$ & $0.27776000 \mathrm{E}+05$ \\
\hline 3.2 & $0.62961235 \mathrm{E}+09$ & $0.62964160 \mathrm{E}+09$ & $0.29248000 \mathrm{E}+05$ \\
\hline 3.5 & $0.66211213 \mathrm{E}+09$ & $0.66214278 \mathrm{E}+09$ & $0.30656000 \mathrm{E}+05$ \\
\hline 3.8 & $0.69243584 \mathrm{E}+09$ & $0.69246771 \mathrm{E}+09$ & $0.31872000 \mathrm{E}+05$ \\
\hline 4.1 & $0.72072896 \mathrm{E}+09$ & $0.72076198 \mathrm{E}+09$ & $0.33024000 \mathrm{E}+05$ \\
\hline 4.3 & $0.74712755 \mathrm{E}+09$ & $0.74716166 \mathrm{E}+09$ & $0.34112000 \mathrm{E}+05$ \\
\hline 4.6 & $0.77175834 \mathrm{E}+09$ & $0.77179347 \mathrm{E}+09$ & $0.35136000 \mathrm{E}+05$ \\
\hline 4.9 & $0.79473990 \mathrm{E}+09$ & $0.79477600 \mathrm{E}+09$ & $0.36096000 \mathrm{E}+05$ \\
\hline 5.1 & $0.81618259 \mathrm{E}+09$ & $0.81621952 \mathrm{E}+09$ & $0.36928000 \mathrm{E}+05$ \\
\hline 5.4 & $0.83618944 \mathrm{E}+09$ & $0.83622720 \mathrm{E}+09$ & $0.37760000 \mathrm{E}+05$ \\
\hline 5.7 & $0.85485651 \mathrm{E}+09$ & $0.85489510 \mathrm{E}+09$ & $0.38592000 \mathrm{E}+05$ \\
\hline 5.9 & $0.87227360 \mathrm{E}+09$ & $0.87231296 \mathrm{E}+09$ & $0.39360000 \mathrm{E}+05$ \\
\hline 6.2 & $0.88852448 \mathrm{E}+09$ & $0.88856454 \mathrm{E}+09$ & $0.40064000 \mathrm{E}+05$ \\
\hline 6.5 & $0.90368698 \mathrm{E}+09$ & $0.90372781 \mathrm{E}+09$ & $0.40832000 \mathrm{E}+05$ \\
\hline 6.8 & $0.91783430 \mathrm{E}+09$ & $0.91787584 \mathrm{E}+09$ & $0.41536000 \mathrm{E}+05$ \\
\hline 7.0 & $0.93103411 \mathrm{E}+09$ & $0.93107635 \mathrm{E}+09$ & $0.42240000 \mathrm{E}+05$ \\
\hline 7.3 & $0.94335002 \mathrm{E}+09$ & $0.94339296 \mathrm{E}+09$ & $0.42944000 \mathrm{E}+05$ \\
\hline 7.6 & $0.95484115 \mathrm{E}+09$ & $0.95488486 \mathrm{E}+09$ & $0.43712000 \mathrm{E}+05$ \\
\hline 7.8 & $0.96556282 \mathrm{E}+09$ & $0.96560730 \mathrm{E}+09$ & $0.44480000 \mathrm{E}+05$ \\
\hline 8.1 & $0.97556634 \mathrm{E}+09$ & $0.97561165 \mathrm{E}+09$ & $0.45312000 \mathrm{E}+05$ \\
\hline 8.4 & $0.98490010 \mathrm{E}+09$ & $0.98494624 \mathrm{E}+09$ & $0.46144000 \mathrm{E}+05$ \\
\hline 8.6 & $0.99360858 \mathrm{E}+09$ & $0.99365568 \mathrm{E}+09$ & $0.47104000 \mathrm{E}+05$ \\
\hline 8.9 & $0.10017338 \mathrm{E}+10$ & $0.10017819 \mathrm{E}+10$ & $0.48064000 \mathrm{E}+05$ \\
\hline 9.2 & $0.10093149 \mathrm{E}+10$ & $0.10093640 \mathrm{E}+10$ & $0.49088000 \mathrm{E}+05$ \\
\hline 9.5 & $0.10163882 \mathrm{E}+10$ & $0.10164384 \mathrm{E}+10$ & $0.50240000 \mathrm{E}+05$ \\
\hline 9.7 & $0.10229876 \mathrm{E}+10$ & $0.10230390 \mathrm{E}+10$ & $0.51456000 \mathrm{E}+05$ \\
\hline 10.0 & $0.10291450 \mathrm{E}+10$ & $0.10291978 \mathrm{E}+10$ & $0.52800000 \mathrm{E}+05$ \\
\hline 10.3 & $0.10348898 \mathrm{E}+10$ & $0.10349440 \mathrm{E}+10$ & $0.54208000 \mathrm{E}+05$ \\
\hline 10.5 & $0.10402498 \mathrm{E}+10$ & $0.10403055 \mathrm{E}+10$ & $0.55744000 \mathrm{E}+05$ \\
\hline 10.8 & $0.10452506 \mathrm{E}+10$ & $0.10453080 \mathrm{E}+10$ & $0.57472000 \mathrm{E}+05$ \\
\hline 11.1 & $0.10499162 \mathrm{E}+10$ & $0.10499755 \mathrm{E}+10$ & $0.59328000 \mathrm{E}+05$ \\
\hline 11.3 & $0.10542692 \mathrm{E}+10$ & $0.10543305 \mathrm{E}+10$ & $0.61312000 \mathrm{E}+05$ \\
\hline 11.6 & $0.10583304 \mathrm{E}+10$ & $0.10583939 E+10$ & $0.63488000 \mathrm{E}+05$ \\
\hline 11.9 & $0.10621193 \mathrm{E}+10$ & $0.10621851 \mathrm{E}+10$ & $0.65792000 \mathrm{E}+05$ \\
\hline 12.2 & $0.10656540 \mathrm{E}+10$ & $0.10657224 \mathrm{E}+10$ & $0.68416000 \mathrm{E}+05$ \\
\hline 12.4 & $0.10689518 \mathrm{E}+10$ & $0.10690230 \mathrm{E}+10$ & $0.71168000 \mathrm{E}+05$ \\
\hline 12.7 & $0.10720284 \mathrm{E}+10$ & $0.10721025 \mathrm{E}+10$ & $0.74112000 \mathrm{E}+05$ \\
\hline 13.0 & $0.10748984 \mathrm{E}+10$ & $0.10749757 \mathrm{E}+10$ & $0.77312000 \mathrm{E}+05$ \\
\hline 13.2 & $0.10775758 \mathrm{E}+10$ & $0.10776567 \mathrm{E}+10$ & $0.80896000 \mathrm{E}+05$ \\
\hline 13.5 & $0.10800735 \mathrm{E}+10$ & $0.10801581 \mathrm{E}+10$ & $0.84608000 \mathrm{E}+05$ \\
\hline 13.8 & $0.10824033 \mathrm{E}+10$ & $0.10824920 \mathrm{E}+10$ & $0.88704000 \mathrm{E}+05$ \\
\hline 14.0 & $0.10845765 \mathrm{E}+10$ & $0.10846696 \mathrm{E}+10$ & $0.93056000 \mathrm{E}+05$ \\
\hline 14.3 & $0.10866036 \mathrm{E}+10$ & $0.10867013 \mathrm{E}+10$ & $0.97664000 \mathrm{E}+05$ \\
\hline
\end{tabular}


Estimation Of Build-Up Of Nuclide During Irradiation By Solving Ode Through Adomian

\begin{tabular}{|c|c|c|c|}
\hline 14.6 & $0.10884945 \mathrm{E}+10$ & $0.10885971 \mathrm{E}+10$ & $0.10265600 \mathrm{E}+06$ \\
\hline 14.9 & $0.10902579 \mathrm{E}+10$ & $0.10903660 \mathrm{E}+10$ & $0.10803200 \mathrm{E}+06$ \\
\hline 15.1 & $0.10919026 \mathrm{E}+10$ & $0.10920163 \mathrm{E}+10$ & $0.11366400 \mathrm{E}+06$ \\
\hline 15.4 & $0.10934363 \mathrm{E}+10$ & $0.10935561 \mathrm{E}+10$ & $0.11980800 \mathrm{E}+06$ \\
\hline 15.7 & $0.10948667 \mathrm{E}+10$ & $0.10949929 \mathrm{E}+10$ & $0.12620800 \mathrm{E}+06$ \\
\hline 15.9 & $0.10962002 \mathrm{E}+10$ & $0.10963333 \mathrm{E}+10$ & $0.13312000 \mathrm{E}+06$ \\
\hline 16.2 & $0.10974436 \mathrm{E}+10$ & $0.10975841 \mathrm{E}+10$ & $0.14054400 \mathrm{E}+06$ \\
\hline 16.5 & $0.10986028 \mathrm{E}+10$ & $0.10987511 \mathrm{E}+10$ & $0.14835200 \mathrm{E}+06$ \\
\hline 16.7 & $0.10996833 \mathrm{E}+10$ & $0.10998400 \mathrm{E}+10$ & $0.15667200 \mathrm{E}+06$ \\
\hline 17.0 & $0.11006904 \mathrm{E}+10$ & $0.11008559 \mathrm{E}+10$ & $0.16550400 \mathrm{E}+06$ \\
\hline 17.3 & $0.11016292 \mathrm{E}+10$ & $0.11018039 \mathrm{E}+10$ & $0.17472000 \mathrm{E}+06$ \\
\hline 17.6 & $0.11025034 \mathrm{E}+10$ & $0.11026883 \mathrm{E}+10$ & $0.18483200 \mathrm{E}+06$ \\
\hline 17.8 & $0.11033185 \mathrm{E}+10$ & $0.11035136 \mathrm{E}+10$ & $0.19507200 \mathrm{E}+06$ \\
\hline 18.1 & $0.11040773 \mathrm{E}+10$ & $0.11042835 \mathrm{E}+10$ & $0.20620800 \mathrm{E}+06$ \\
\hline 18.4 & $0.11047837 \mathrm{E}+10$ & $0.11050019 \mathrm{E}+10$ & $0.21811200 \mathrm{E}+06$ \\
\hline 18.6 & $0.11054418 \mathrm{E}+10$ & $0.11056722 \mathrm{E}+10$ & $0.23040000 \mathrm{E}+06$ \\
\hline 18.9 & $0.11060545 \mathrm{E}+10$ & $0.11062976 \mathrm{E}+10$ & $0.24307200 \mathrm{E}+06$ \\
\hline 19.2 & $0.11066243 \mathrm{E}+10$ & $0.11068812 \mathrm{E}+10$ & $0.25689600 \mathrm{E}+06$ \\
\hline 19.4 & $0.11071543 \mathrm{E}+10$ & $0.11074257 \mathrm{E}+10$ & $0.27136000 \mathrm{E}+06$ \\
\hline 19.7 & $0.11076472 \mathrm{E}+10$ & $0.11079337 \mathrm{E}+10$ & $0.28646400 \mathrm{E}+06$ \\
\hline 20.0 & $0.11081052 \mathrm{E}+10$ & $0.11084076 \mathrm{E}+10$ & $0.30233600 \mathrm{E}+06$ \\
\hline 20.3 & $0.11085308 \mathrm{E}+10$ & $0.11088499 \mathrm{E}+10$ & $0.31910400 \mathrm{E}+06$ \\
\hline 20.5 & $0.11089257 \mathrm{E}+10$ & $0.11092625 \mathrm{E}+10$ & $0.33676800 \mathrm{E}+06$ \\
\hline 20.8 & $0.11092927 \mathrm{E}+10$ & $0.11096475 \mathrm{E}+10$ & $0.35481600 \mathrm{E}+06$ \\
\hline 21.1 & $0.11096325 \mathrm{E}+10$ & $0.11100068 \mathrm{E}+10$ & $0.37427200 \mathrm{E}+06$ \\
\hline 21.3 & $0.11099475 \mathrm{E}+10$ & $0.11103419 \mathrm{E}+10$ & $0.39436800 \mathrm{E}+06$ \\
\hline 21.6 & $0.11102392 \mathrm{E}+10$ & $0.11106546 \mathrm{E}+10$ & $0.41536000 \mathrm{E}+06$ \\
\hline 21.9 & $0.11105089 \mathrm{E}+10$ & $0.11109464 \mathrm{E}+10$ & $0.43750400 \mathrm{E}+06$ \\
\hline 22.1 & $0.11107578 \mathrm{E}+10$ & $0.11112187 \mathrm{E}+10$ & $0.46092800 \mathrm{E}+06$ \\
\hline 22.4 & $0.11109882 \mathrm{E}+10$ & $0.11114726 \mathrm{E}+10$ & $0.48448000 \mathrm{E}+06$ \\
\hline 22.7 & $0.11111997 \mathrm{E}+10$ & $0.11117097 \mathrm{E}+10$ & $0.50995200 \mathrm{E}+06$ \\
\hline 23.0 & $0.11113944 \mathrm{E}+10$ & $0.11119308 \mathrm{E}+10$ & $0.53632000 \mathrm{E}+06$ \\
\hline 23.2 & $0.11115736 \mathrm{E}+10$ & $0.11121371 \mathrm{E}+10$ & $0.56345600 \mathrm{E}+06$ \\
\hline 23.5 & $0.11117376 \mathrm{E}+10$ & $0.11123296 \mathrm{E}+10$ & $0.59200000 \mathrm{E}+06$ \\
\hline 23.8 & $0.11118876 \mathrm{E}+10$ & $0.11125093 \mathrm{E}+10$ & $0.62169600 \mathrm{E}+06$ \\
\hline 24.0 & $0.11120241 \mathrm{E}+10$ & $0.11126769 \mathrm{E}+10$ & $0.65280000 \mathrm{E}+06$ \\
\hline 24.3 & $0.11121481 \mathrm{E}+10$ & $0.11128332 \mathrm{E}+10$ & $0.68505600 \mathrm{E}+06$ \\
\hline 24.6 & $0.11122605 \mathrm{E}+10$ & $0.11129792 \mathrm{E}+10$ & $0.71872000 \mathrm{E}+06$ \\
\hline 24.8 & $0.11123619 \mathrm{E}+10$ & $0.11131153 \mathrm{E}+10$ & $0.75340800 \mathrm{E}+06$ \\
\hline 25.1 & $0.11124526 \mathrm{E}+10$ & $0.11132422 \mathrm{E}+10$ & $0.78963200 \mathrm{E}+06$ \\
\hline 25.4 & $0.11125338 \mathrm{E}+10$ & $0.11133608 \mathrm{E}+10$ & $0.82700800 \mathrm{E}+06$ \\
\hline 25.7 & $0.11126048 \mathrm{E}+10$ & $0.11134714 \mathrm{E}+10$ & $0.86656000 \mathrm{E}+06$ \\
\hline 25.9 & $0.11126676 \mathrm{E}+10$ & $0.11135745 \mathrm{E}+10$ & $0.90688000 \mathrm{E}+06$ \\
\hline 26.2 & $0.11127224 \mathrm{E}+10$ & $0.11136708 \mathrm{E}+10$ & $0.94835200 \mathrm{E}+06$ \\
\hline 26.5 & $0.11127684 \mathrm{E}+10$ & $0.11137606 \mathrm{E}+10$ & $0.99225600 \mathrm{E}+06$ \\
\hline 26.7 & $0.11128072 \mathrm{E}+10$ & $0.11138444 \mathrm{E}+10$ & $0.10371840 \mathrm{E}+07$ \\
\hline 27.0 & $0.11128381 \mathrm{E}+10$ & $0.11139226 \mathrm{E}+10$ & $0.10844160 \mathrm{E}+07$ \\
\hline
\end{tabular}

Table 5: Result for a day at a time when Utah's assumptions are ignored.

\begin{tabular}{|c|c|c|c|}
\hline $\mathbf{X}$ & ADOMIAN & ACTUAL & ERROR \\
\hline 0.0 & $0.00000000 \mathrm{E}+00$ & $0.00000000 \mathrm{E}+00$ & $0.00000000 \mathrm{E}+00$ \\
\hline 1.0 & $0.25242611 \mathrm{E}+09$ & $0.25243843 \mathrm{E}+09$ & $0.12320000 \mathrm{E}+05$ \\
\hline 2.0 & $0.44770358 \mathrm{E}+09$ & $0.44772493 \mathrm{E}+09$ & $0.21344000 \mathrm{E}+05$ \\
\hline 3.0 & $0.59877056 \mathrm{E}+09$ & $0.59879853 \mathrm{E}+09$ & $0.27968000 \mathrm{E}+05$ \\
\hline 4.0 & $0.71563635 \mathrm{E}+09$ & $0.71566918 \mathrm{E}+09$ & $0.32832000 \mathrm{E}+05$ \\
\hline 5.0 & $0.80604378 \mathrm{E}+09$ & $0.80608032 \mathrm{E}+09$ & $0.36544000 \mathrm{E}+05$ \\
\hline 6.0 & $0.87598291 \mathrm{E}+09$ & $0.87602240 \mathrm{E}+09$ & $0.39488000 \mathrm{E}+05$ \\
\hline 7.0 & $0.93008736 \mathrm{E}+09$ & $0.93012954 \mathrm{E}+09$ & $0.42176000 \mathrm{E}+05$ \\
\hline 8.0 & $0.97194195 \mathrm{E}+09$ & $0.97198694 \mathrm{E}+09$ & $0.44992000 \mathrm{E}+05$ \\
\hline 9.0 & $0.10043194 \mathrm{E}+10$ & $0.10043678 \mathrm{E}+10$ & $0.48384000 \mathrm{E}+05$ \\
\hline 10.0 & $0.10293649 \mathrm{E}+10$ & $0.10294177 \mathrm{E}+10$ & $0.52800000 \mathrm{E}+05$ \\
\hline 11.0 & $0.10487374 \mathrm{E}+10$ & $0.10487962 \mathrm{E}+10$ & $0.58816000 \mathrm{E}+05$ \\
\hline 12.0 & $0.10637204 \mathrm{E}+10$ & $0.10637875 \mathrm{E}+10$ & $0.67008000 \mathrm{E}+05$ \\
\hline
\end{tabular}




\begin{tabular}{|l|l|l|l|}
\hline 13.0 & $0.10753068 \mathrm{E}+10$ & $0.10753847 \mathrm{E}+10$ & $0.77952000 \mathrm{E}+05$ \\
\hline 14.0 & $0.10842641 \mathrm{E}+10$ & $0.10843564 \mathrm{E}+10$ & $0.92288000 \mathrm{E}+05$ \\
\hline 15.0 & $0.10911857 \mathrm{E}+10$ & $0.10912968 \mathrm{E}+10$ & $0.11110400 \mathrm{E}+06$ \\
\hline 16.0 & $0.10965308 \mathrm{E}+10$ & $0.10966659 \mathrm{E}+10$ & $0.13504000 \mathrm{E}+06$ \\
\hline 17.0 & $0.11006542 \mathrm{E}+10$ & $0.11008195 \mathrm{E}+10$ & $0.16524800 \mathrm{E}+06$ \\
\hline 18.0 & $0.11038303 \mathrm{E}+10$ & $0.11040328 \mathrm{E}+10$ & $0.20249600 \mathrm{E}+06$ \\
\hline 19.0 & $0.11062702 \mathrm{E}+10$ & $0.11065184 \mathrm{E}+10$ & $0.24819200 \mathrm{E}+06$ \\
\hline 20.0 & $0.11081379 \mathrm{E}+10$ & $0.11084413 \mathrm{E}+10$ & $0.30348800 \mathrm{E}+06$ \\
\hline 21.0 & $0.11095590 \mathrm{E}+10$ & $0.11099290 \mathrm{E}+10$ & $0.36992000 \mathrm{E}+06$ \\
\hline 22.0 & $0.11106312 \mathrm{E}+10$ & $0.11110798 \mathrm{E}+10$ & $0.44864000 \mathrm{E}+06$ \\
\hline 23.0 & $0.11114286 \mathrm{E}+10$ & $0.11119700 \mathrm{E}+10$ & $0.54144000 \mathrm{E}+06$ \\
\hline 24.0 & $0.11120097 \mathrm{E}+10$ & $0.11126588 \mathrm{E}+10$ & $0.64908800 \mathrm{E}+06$ \\
\hline 25.0 & $0.11124168 \mathrm{E}+10$ & $0.11131916 \mathrm{E}+10$ & $0.77478400 \mathrm{E}+06$ \\
\hline 26.0 & $0.11126845 \mathrm{E}+10$ & $0.11136037 \mathrm{E}+10$ & $0.91916800 \mathrm{E}+06$ \\
\hline 27.0 & $0.11128381 \mathrm{E}+10$ & $0.11139226 \mathrm{E}+10$ & $0.10844160 \mathrm{E}+07$ \\
\hline 28.0 & $0.11128966 \mathrm{E}+10$ & $0.11141692 \mathrm{E}+10$ & $0.12725760 \mathrm{E}+07$ \\
\hline 29.0 & $0.11128737 \mathrm{E}+10$ & $0.11143601 \mathrm{E}+10$ & $0.14863360 \mathrm{E}+07$ \\
\hline 30.0 & $0.11127805 \mathrm{E}+10$ & $0.11145076 \mathrm{E}+10$ & $0.17271040 \mathrm{E}+07$ \\
\hline
\end{tabular}

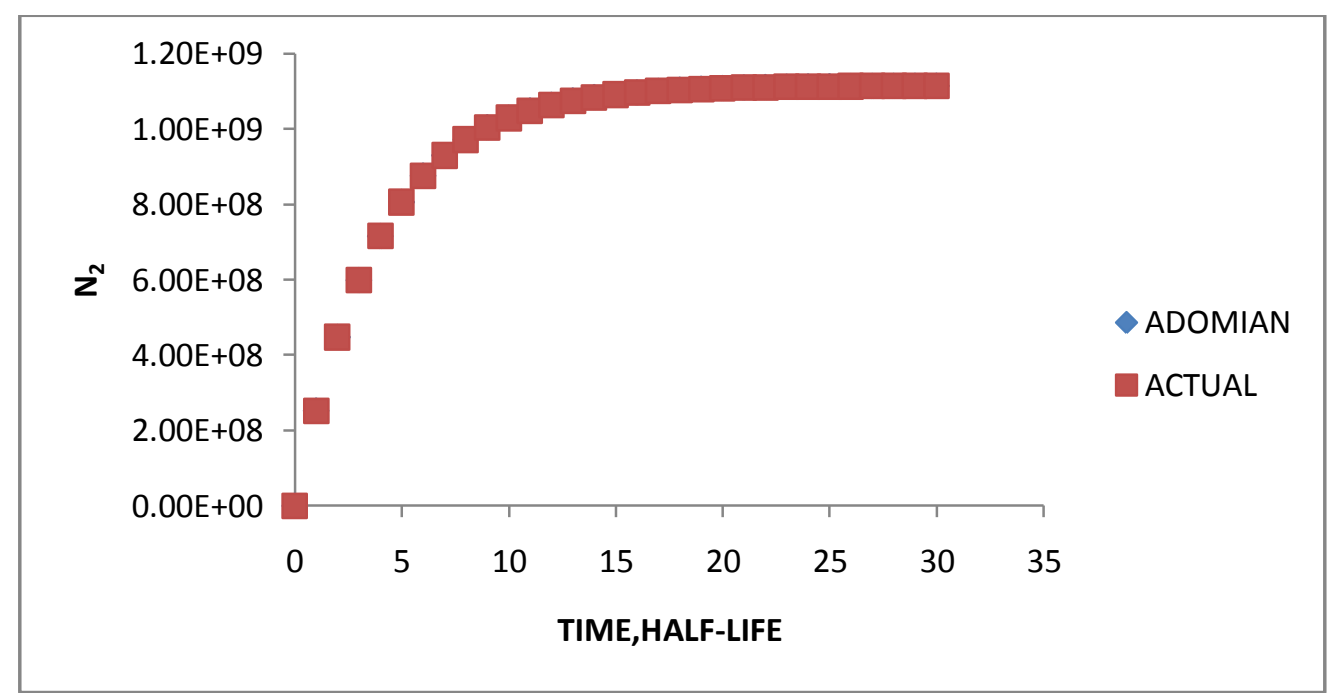

Fig 1. Actual values of $\mathrm{N}_{2}$, its Adomian approximation for a day at a time we used Utah's assumptions.

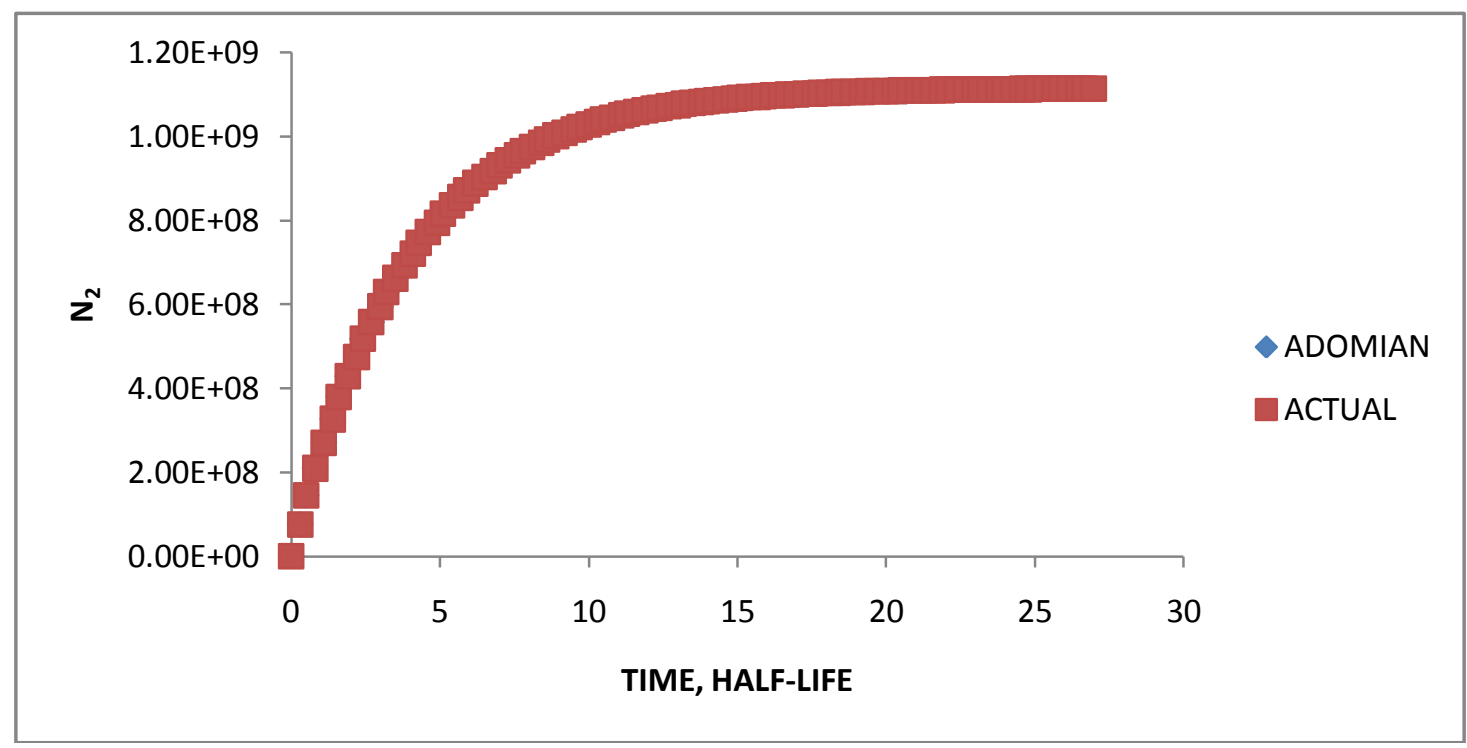

Fig 2. Actual values of $\mathrm{N}_{2}$, its Adomian approximation when we used Utah's assumptions for fractionated days (2.7 days). 


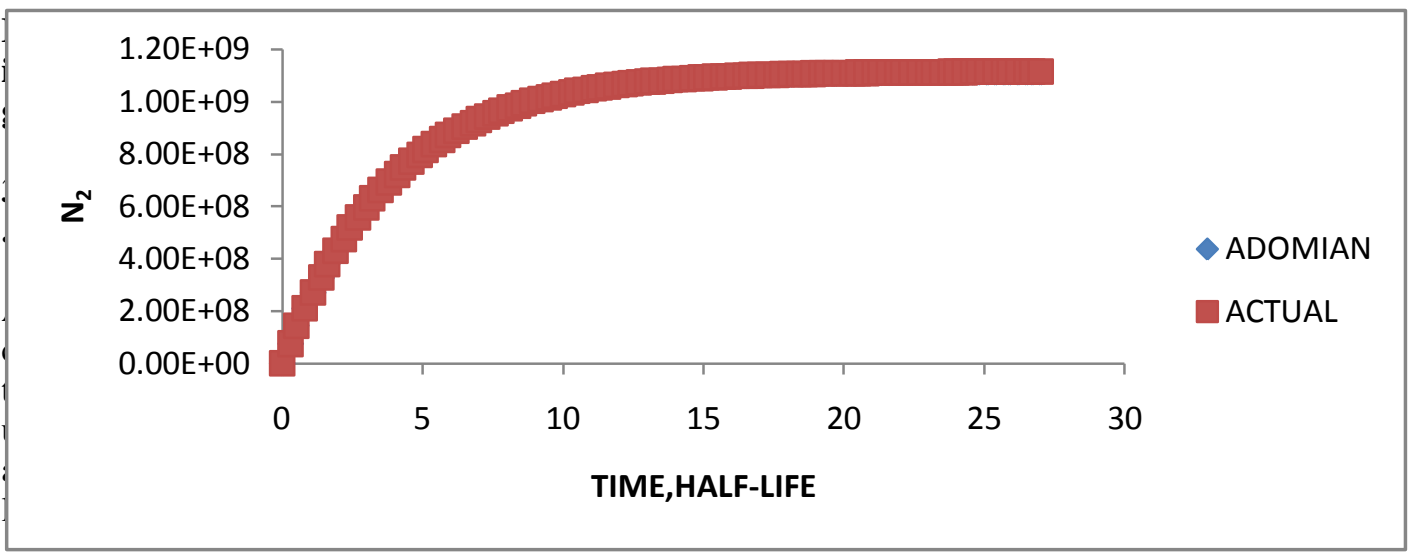

values of $\mathrm{N}_{2}$, its Adomian approximation when Utah's assumptions are ignored for fractionated days (2.7 days).

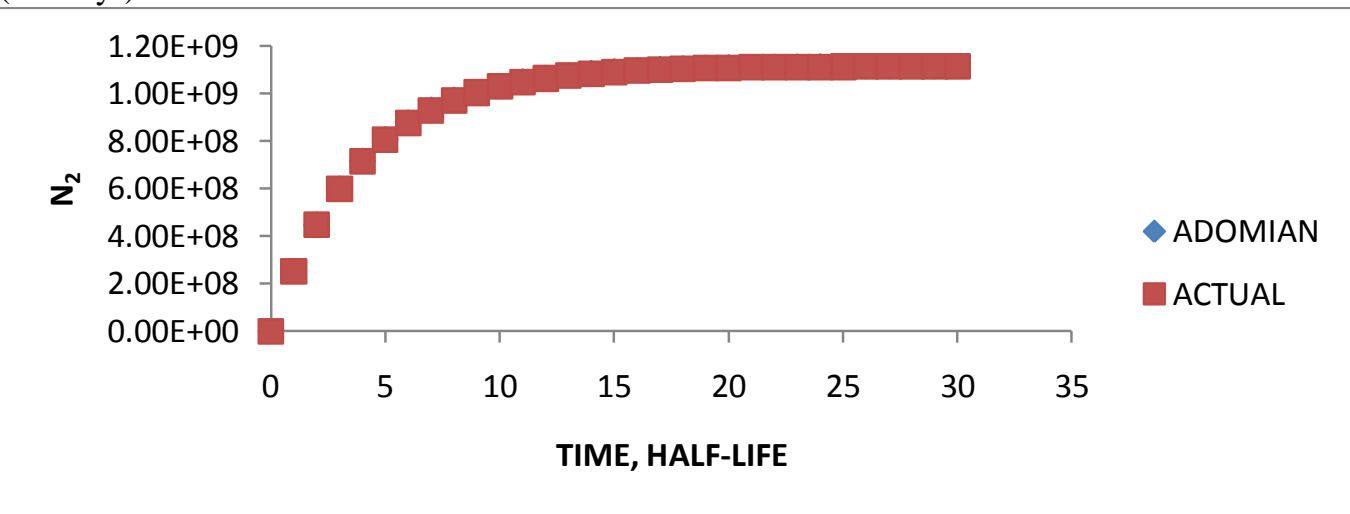

Fig 4. Actual values of $\mathrm{N}_{2}$, its Adomian approximation for a day at a time Utah's assumptions are ignored.

\section{Conclusion}

We presented a method used to obtain analytic approximate solution of FODE of NAA equation for the build-up of nuclide $\left(N_{2}\right)$ during irradiation by using ADM. The absolute error, actual value( by Utah) and numerical results are presented and compared with one another in tables and figures for some values of $N_{2}$ at varied $t$. ADM shows good agreement with Utah actual solution even over large terms and converge fast to actual. This shows that ADM is reliable, powerful, promising and efficient method to obtain accurate solutions.

\section{References}

[1] Abdusalam, H.A and Alabdulatif, M (2006). Exact and numerical solutions of the telegraph model of dispersive variability. Applied Numerical Mathematics. Far East. J. of Dynamic System 8(1):65.

[2] Adomian, G (1994).Solving frontier problem of Physics. The decomposition method (Boston, M.A: Kluwer Academic).

[3] Antoaneta, E, Ana, P, Carmo, F and Alina, B (2011). EDXRF and INAA analysis of soils in the vicinity of a metallurgical plant. Rom.Journ Phys. 56(7-8): 993-1000.

[4] Ellis, D.V (1987).Well logging for Earth scientists. 1st Ed. Elsevier: 523.

[5] Ellis, D.V and Singer, J.M (2008). Well logging for Earth Scientists. $2^{\text {nd }}$ Ed. Springer: 629.

[6] Essiett, A.A, Uwah, E.J and Uwak, S.O (2011). Elemental analysis of soil around Ikot Abasi, aluminum smelter plant, Nigeria by Instrumental Activation Analysis (INAA). Archives of Applied Science Research 3(1): 25-32

[7] Evans, D.J and Raslan K.R (2004). The Adomian decomposition method for solving delay differential equation. International Journal of Computer Mathematics 0(0):1-6.

[8] Hassan, A.M (2008). Modern trends in neutron activation analysis. Application to some African Environmental samples. Proceedings of the $3^{\text {rd }}$ Environmental Physics Conference, 19-23, 2008, Aswan, Egypt. 27-40.

[9] Ioan,S, Constantin, C, mihai, T, Sorana, D.B and Lorentz, J (2008). Analysis of soil heavy metal pollution in central Transylvania. Int. J. Mol. Sci. 9: 434-453.

[10] Kogo, B.E, Gajere, E.N, Ogunmola, J.K and Ogbole, J.O (2009). Neutron activation analysis of soil samples from different parts of Abuja metropolis. Middle-East Journal of Scientific research 494): 254-262.

[11] Molnar, Z (2012). Neutron activation analysis, http://serc.carleton.edu/18404/ retrieved in 2012.

[12] Nargolwalla, S.S and Przybylowicz, E.P (1973). Activation analysis with neutron generator, V39, monograph series, chemical analysis John Wiley\& son: 662.

[13] Nelson, E (2012). Instrumental neutron activation analysis (INAA). University of Massachusetts Lowell; http:// www. Neutron activation analysis, retrieved in 2012.

[14] Utah (2012). Ch_3_neutron_activation, www.nuclear.utah.edu retrieved in 2012

[15] Waring, C, Falahat, S and Watt, G (2011). What can you measure with neutron activation analysis. The Second Global Workshop on Proximal Soil Sensing-Montreal 2011: 120-123.

[16] Wazwaz, A.M (2002). Partial differential equations: Methods and applications, Balkema Publishers. The Netherlands. 